Memorias del VII Encuentro Nacional de Experiencias en la Enseñanza de la Biología y la Educación Ambiental y 11 Congreso Nacional de Investigación en la Enseñanza de la Biología

\title{
REFLEXIÓNES EN TORNO A LA PROBLEMÁTICA DE INTRODUCCIÓN DE PLANTAS EXÓTICAS PRESENTES EN MI COLEGIO, UNA OPORTUNIDAD EN LA CONSTRUCCIÓN DEL CONCEPTO DE BIODIVERSIDAD
}

\author{
REFLECTIONS ON THE PROBLEM OF INTRODUCTION OF EXOTIC PLANTS \\ FOUND IN MY SCHOOL AS AN ITEM CONSTRUCTION OF BIODIVERSITY
}

\author{
Raquel Sofía Soto Soto ${ }^{1}$
}

\section{Resumen}

La biodiversidad comprendida como la variabilidad de seres vivos, y ecosistemas organizados en los niveles genéticos, de especies y de ecosistemas, ha disminuido de manera acelerada debido a acciones propias de la intervención antropica como la introducción de especies de plantas exóticas que generan la pérdida de especies nativas y pérdida las características propias de los ecosistemas por tal motivo es importante generar en los estudiantes el interés por el reconocimiento de la biodiversidad y las causas de la pérdida en su entorno. De acuerdo a ello se construye la propuesta didáctica ¿Biodiversidad en mi entorno?, que se implementó bajo el enfoque de Enseñanza para la Comprensión, en la IED La Estancia San Isidro Labrador del grado 901 comprendido por estudiantes entre los 12 y 17 años. Durante la implementación de esta propuesta didáctica, los estudiantes por medio del reconocimiento de las plantas nativas y exóticas presentes en su entorno (colegio), y el efecto que ejercen estas plantas sobre el ecosistema, logran acercamientos a la construcción del concepto de especie; todo ello permitió demostrar los avances que lograron estudiantes en la dimensión de contenidos donde ya reconocen la biodiversidad como la variabilidad de organismos y las funciones que ejercen los mismo con el medio y en cuanto a la dimensión de formas de comunicación, ahora adquieren la intención estética y oral para comunicar lo aprendido sobre la biodiversidad por medio de distintos géneros y símbolos.

Palabras Clave: Entorno, ecosistemas, plantas nativas, contenido, formas de comunicación.

\footnotetext{
${ }^{1}$ Estudiante del Proyecto curricular de Licenciatura en Biología de la Universidad Distrital Francisco José de Caldas. Docente practicante I.E.D. La Estancia San Isidro Labrador. Integrante del semillero BER (Biología Enseñanza y Realidades) Correo: rssotos@correo.udistrital.edu.co
} 
Memorias del VII Encuentro Nacional de Experiencias en la Enseñanza de la Biología y la Educación Ambiental y 11 Congreso Nacional de Investigación en la Enseñanza de la Biología

\section{Abstract}

The biodiversity is including as the variability of living organisms, and ecosystems, this organized in genetic, species and ecosystem levels, this has declined rapidly for reasons related to the entropic intervention such as the introduction of exotic species plant in the ecosystem, which generate the loss of native species and the characteristics of an ecosystem, for this reason it is important to instill in students the interest in the recognition of biodiversity and the causes of loss in their environment, It was built for the didactic proposal, Biodiversity in my environment?, implemented under the approach of teaching for understanding, IED Estancia San Isidro Labrador comprised 901 degree students between 12 and 17 years old. During the implementation of this proposal didactic, students recognized native and exotic plants present in their environment (college), and the effect they have these plants on the ecosystem, all this helped to show the students progress seen in the dimension of content which already recognize biodiversity as the variability among living organisms and functions exercised by the same means and about the dimension of communication forms, now acquire aesthetics and oral intend to communicate biodiversity learned through different genres and symbols.

Key Words: Environment, ecosystems, native plants, content, forms of communication.

\section{Introducción}

En esta primera parte del artículo, se presentan los marcos teóricos sobre la Enseñanza para la Comprensión, el marco disciplinar y didáctico correspondiente al concepto de biodiversidad, y el objetivo principal del articulo.

Enseñanza para la Comprensión como enfoque pedagógico según Stone (1999), parte de la definición de comprensión como la capacidad de remitirse al conocimiento para resolver problemas desempeñándose de manera flexible; es decir, demostrando destrezas para explicar, justificar, explorar, vincular y aplicar de maneras que van más allá del conocimiento y la habilidad rutinaria, de acuerdo con Perkins, 1999, citado por De Longhi (2006).

La comprensión que los estudiantes logran en el aula se debe evidenciar por medio de 4 dimensiones que son contenidos, métodos, propósitos y formas de comunicación, cada uno de estos a su vez opera diferentes niveles de profundidad denominados como ingenuo, principiante, aprendiz y maestría. En el nivel ingenuo, los estudiantes no ven la relación entre lo que aprenden en la escuela y su vida cotidiana. En el nivel principiante, empiezan destacando algunos conceptos ó ideas disciplinarios, establecen simples conexiones entre ellas, a menudo ensayadas, y los procedimientos son realizados mecánicamente paso por paso subyugado a la autoridad del docente. En el nivel de aprendiz, demuestran 
Memorias del VII Encuentro Nacional de Experiencias en la Enseñanza de la Biología y la Educación Ambiental y 11 Congreso Nacional de Investigación en la Enseñanza de la Biología

un uso flexible de conceptos o ideas de la disciplina, esto con apoyo del docente, también se puede observar la relación entre conocimiento disciplinario y vida cotidiana, asimismo expresan y comunican el conocimiento de manera adecuada. Y en el Nivel de maestría, el conocimiento es expresado y comunicado a otros de manera creativa, Los estudiantes en este nivel a menudo van más allá, demostrando comprensión disciplinaria: pueden reflejar la conciencia crítica acerca de la construcción del conocimiento en el dominio. Una cualidad importante del desempeño de comprensión del ejemplo es su naturaleza integrada y crítica. (Stone 1999)

Por tanto el objetivo de este artículo es demostrar los niveles de comprensión alcanzados por los estudiantes de grado 901, de la IED la estancia San Isidro labrador, en las dimensiones de contenidos y formas de comunicación entorno al concepto de biodiversidad. Esto en base a una experiencia pedagógica denominada ¿Biodiversidad en mi entorno? Donde los estudiantes reconocen de la biodiversidad en su entorno, identifican el problema de introducción de plantas exóticas en su colegio y el aportan soluciones al mismo.

Para ello se tiene en cuenta que dentro la de la biología se ha dado muchas definiciones de biodiversidad muy semejantes a la siguiente:

\begin{abstract}
"La biología ha definido la biodiversidad como la variedad y variabilidad de los seres vivos y de los ecosistemas que integran. Los componentes de la diversidad biológica se organizan en tres niveles: el de los genes, que constituyen las bases moleculares de la herencia; el de las especies, que son conjuntos de organismos afines capaces de reproducirse entre sí; y el de los ecosistemas, que son complejos funcionales formados por los organismos y el medio físico en el que habitan" (Crisci, 2006 p.1).
\end{abstract}

Por otro lado, Rozzi, R., Feinsinger P., Massardo, F. \& Primack, R. (2001). complementan la definición de variedad de seres vivos aportando el hecho de que los complejos ecológicos donde se encuentran, también hacen parte de la biodiversidad y además de estar organizadas en los tres niveles mencionados anteriormente, se distinguen tres atributos que son estructura función y composición (Noss 1990). El atributo de estructura considera la posición y organización de los componentes del ecosistema, el de composición hace referencia a los niveles jerárquicos en que se encuentra organizada la biodiversidad y la función a los procesos (ecológicos, bioquímicos y evolutivos) que cumplen estos componentes biológicos.

Actualmente existen varios problemas que afectan la biodiversidad a nivel de especie, entre ellos la introducción de especies exóticas que es una de las principales causas de extinción, para ello una medida de conservación de acuerdo con Martínez (2002) ha sido la introducción de más individuos, razas ó 
Memorias del VII Encuentro Nacional de Experiencias en la Enseñanza de la Biología y la Educación Ambiental y 11 Congreso Nacional de Investigación en la Enseñanza de la Biología

subespecies de la misma especie de ciertos ecosistemas para aumentar el tamaño de las poblaciones en peligro.

Estas precisiones acerca de la enseñanza para la comprensión y el concepto de biodiversidad convergen también en la enseñanza de las ciencias a través de los planteamientos de autores como Bermúdez y De Longhi (2006) que presentan una hipótesis de progresión para abordar los diferentes niveles de complejidad, de ingenuo a maestría sobre los conceptos de Biodiversidad, pérdida y conservación y perturbación. Haciendo énfasis sobre el primer concepto se considera que el nivel ingenuo el estudiante no reconoce la biodiversidad, ó solo existe una visión centrada en el mesocosmos, en el nivel principiante se estima la biodiversidad de acuerdo a la riqueza de especies, en el nivel aprendiz este concepto ya no se determina solo por la riqueza de especies sino también por la abundancia relativa de las mismas y en el nivel maestría se reconoce la biodiversidad como la variabilidad de seres vivos en cualquier ecosistema, su representatividad en el mismo, sus funciones y características, y los tres niveles de manera integrada; a partir de ello los autores exponen los resultados correspondientes a la implementación de una unidad didáctica sobre estos conceptos con estudiantes de $5^{\text {to }}$ grado de ciclo polimodal en una escuela de la provincia Córdoba en Argentina.

De otra manera García, Cano y Rodríguez (2003) explican en un recorrido por la historia la construcción del concepto de biodiversidad, y la visión del hombre sobre el mismo en las diferentes épocas de la historia, aparte de ello demuestra la complejización de las ideas previas que poseen los estudiantes sobre el concepto de biodiversidad, dónde ellos demuestran una visión aditiva sobre la biodiversidad, se reconocen solo los elementos tangibles y muy poco sobre las relaciones entre ellos. Existen entre los estudiantes tendencias a jerarquizar las funciones de los seres vivos en el ecosistema, el tamaño de las especies y la predilección de los medios de comunicación con respecto a una especie. Sobre la relación entre el hombre y la biodiversidad, se tiene como referencia el antropocentrismo, la visión de biodiversidad como recurso, y no como un entramado de relaciones que configuran y modifican el medio que a su vez los modifica en sí mismos incluyendo el aporte que genera el hombre desde su cultura.

\section{Metodología}

Los resultados que se presentan a continuación toman como referente el proceso de investigación que se basa el paradigma interpretativo:

\footnotetext{
"El interés se centra en el estudio de los significados de las acciones humanas y de la vida social (...) Enfatiza la compresión e interpretación de la realidad educativa desde los significados de las personas implicadas en los contextos educativos y estudia sus creencias, intenciones, motivaciones y otras características del proceso educativo no observables directamente ni susceptibles de experimentación" (Arnal \& Latorre, 1992 p. 41).
} 
Bio-grafia Escritos sobre la Biología y su Enseñanza.

Edición Extra-Ordinaria. ISSN 2027-1034 P. p122-132

Memorias del VII Encuentro Nacional de Experiencias en la Enseñanza de la Biología y la Educación Ambiental y 11 Congreso Nacional de Investigación en la Enseñanza de la Biología

Para ello esta investigación presenta los resultados a partir de la toma y análisis de registros como videos y transcripciones de las clases, diario de campo y algunos trabajos realizados por los estudiantes. El proceso realizado en el aula fue el siguiente:

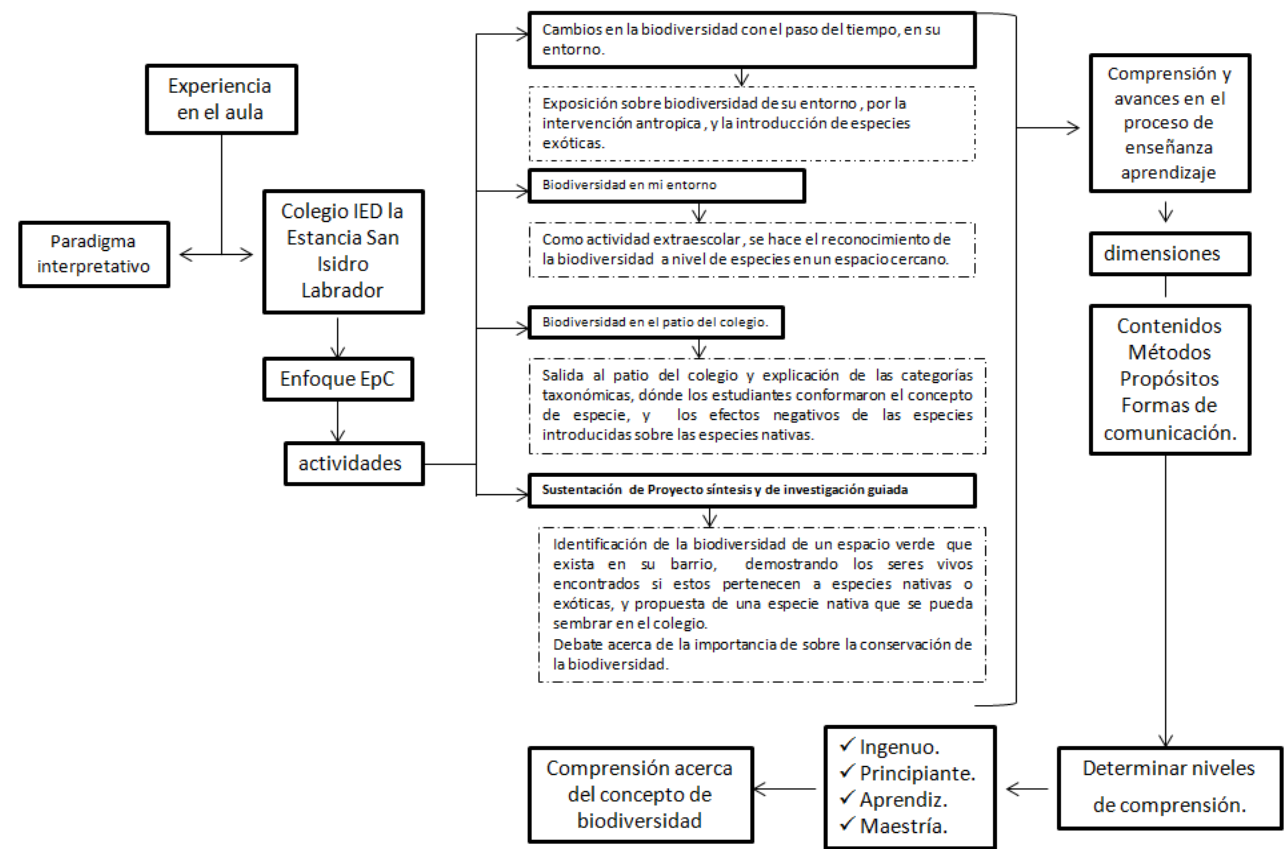

Figura 1 metodología realizada, bajo el enfoque de enseñanza de la EpC.

\begin{tabular}{|c|c|c|}
\hline \multicolumn{3}{|c|}{ Dimensiones y niveles de comprensión } \\
\hline & Contenidos (concepto de biodiversidad) & Formas de comunicación \\
\hline Ingenuo & $\begin{array}{l}\text { No se conoce la biodiversidad en ninguno de los tres niveles, } \\
\text { o por lo menos tiene en cuenta solo el de especie en un } \\
\text { modelo aditivo, tampoco reconoce las causas principales de la } \\
\text { pérdida del mismo ni las acciones que se puedan realizar para } \\
\text { evitarlo. }\end{array}$ & $\begin{array}{l}\text { Las representaciones de los estudiantes } \\
\text { sobre los conceptos en biodiversidad } \\
\text { no son claras, algo que se evidencia en } \\
\text { la dificultad para expresarlo por medio } \\
\text { de diferentes géneros. No hay intención } \\
\text { de comunicación oral y estética. }\end{array}$ \\
\hline Principiante & $\begin{array}{l}\text { El estudiante por medio de la diferenciación de de varios } \\
\text { seres vivos logra algunos acercamientos al concepto de } \\
\text { especie, comienza a asociar la biodiversidad con respecto al } \\
\text { número de especies presentes en un lugar ó ecosistema, Y } \\
\text { hace reconocimiento de algunas causas de la perdida de la } \\
\text { biodiversidad ligadas a la intervención antropica, como la } \\
\text { introducción de especies exóticas, entre otras. }\end{array}$ & $\begin{array}{l}\text { También usan estrategias como } \\
\text { carteleras y dibujos como géneros para } \\
\text { dar cuenta de la complejización de sus } \\
\text { ideas (la biodiversidad presente en su } \\
\text { entorno, la influencia de especies } \\
\text { exóticas sobre especies nativas), } \\
\text { aunque el desarrollo de estos se da de } \\
\text { manera guiada por el docente. Los } \\
\text { estudiantes toman un sistema de } \\
\text { símbolos para explicar en forma de } \\
\text { analogía lo que han aprendido. }\end{array}$ \\
\hline Aprendiz & $\begin{array}{l}\text { Reconoce la influencia de los factores abióticos en el medio y } \\
\text { los ecosistemas, algunos de los procesos que se llevan a } \\
\text { cabo allí aunque de manera muy general, por otro lado se } \\
\text { reconocen los efectos que genera la introducción de especies }\end{array}$ & $\begin{array}{l}\text { Preocupación por exponer sus ideas de } \\
\text { manera clara en público, por medio de } \\
\text { distintos géneros, siendo conscientes de } \\
\text { las pautas que implica la utilización del }\end{array}$ \\
\hline
\end{tabular}


Memorias del VII Encuentro Nacional de Experiencias en la Enseñanza de la Biología y la Educación Ambiental y 11 Congreso Nacional de Investigación en la Enseñanza de la Biología

\begin{tabular}{|c|c|c|}
\hline & $\begin{array}{l}\text { exóticas sobre la biodiversidad de los ecosistemas y la } \\
\text { influencia sobre las especies nativas. }\end{array}$ & $\begin{array}{l}\text { mismo. Al lograr mayor complejidad de } \\
\text { su conocimiento adquieren la capacidad } \\
\text { de expresarse por medio de diferentes } \\
\text { sistemas de símbolos. }\end{array}$ \\
\hline Maestría & $\begin{array}{l}\text { El estudiante logra abarcar el nivel de ecosistemas, reconoce } \\
\text { sus características principales definidas por las especies } \\
\text { propias del mismo y la función de cada componente como } \\
\text { parte de un todo en los procesos de los ecosistemas, } \\
\text { mostrando una comprensión de la biodiversidad como un } \\
\text { modelo de interacción entre las partes. } \\
\text { El estudiante refleja una actitud crítica reflexiva frente a la } \\
\text { problemática la introducción de especies exóticas mostrando } \\
\text { preocupación por proponer soluciones. }\end{array}$ & $\begin{array}{l}\text { Los estudiantes demuestran el dominio } \\
\text { del género por medio de la presentación } \\
\text { de ideas claras, demuestra el uso } \\
\text { flexible de su conocimiento adquirido } \\
\text { por medio de la expresión oral, escrita, } \\
\text { el uso de un lenguaje científico } \\
\text { adecuado. También es capaz de utilizar } \\
\text { de manera integrada estos sistemas de } \\
\text { símbolos. }\end{array}$ \\
\hline
\end{tabular}

Tabla 2. Dimensión de contenidos y formas de comunicación. Los criterios presentados, se basan en los planteamientos realizados por Ángel y De longhi (2006), Martínez (2002), Biox y Gardner (1999) en Stone, Noss (1990) y Rozzi et al. (2001).

\section{Resultados}

Se presentan ahora los aspectos a evaluar en los estudiantes de acuerdo a los niveles de comprensión en las dimensiones de contenidos y formas de comunicación.

Cabe aclarar que se demostrará el proceso de comprensión individual de 6 estudiantes pertenecientes al grado 901, sobre el tópico generativo ¿biodiversidad en mi entorno? y las dimensiones mencionadas anteriormente.

Dimensión de contenido: Gran parte de los estudiantes mostraron avances significativos durante el desarrollo de esta unidad, alcanzado los niveles de aprendiz y algunos acercamientos al nivel de maestría ya que, generaron reflexiones sobre el efecto de la introducción de especies exóticas por medio del reconocimiento de la biodiversidad de su entorno (el patio del colegio), además de ello demostraron preocupación por proponer soluciones ante esta problemática.

Con respecto a las ideas previas, se observa que los 6 estudiantes se ubican en el nivel ingenuo con algunos acercamientos al nivel de aprendiz (estudiantes $1,2,4,5,6)$ debido a que reconocen que es la biodiversidad por medio de ideas como las siguientes: caso 1 "la biodiversidad significa para mi muchas especies ya sea de plantas cosas animales ó clima" caso 4 "la forma de vida que tenemos en nuestro planeta ya que existen millones de "cosas" y cada una de ellas con una característica diferente" caso 5 "es todo Io relacionado con la naturaleza y vida, ejemplo: animales y plantas" estas ideas expresadas por los estudiantes, demuestran que reconocen la biodiversidad en el nivel de especie de acuerdo con los planteamientos de Crisci (2006) y Rozzi et al (2001), por otro lado también la reconocen en el nivel del mesocosmos como un "modelo aditivo, es decir, se reconocen más fácilmente los elementos componentes del medio (o por lo menos los más evidentes y familiares)" (García et al 2003), también se puede observar que desarrollan el concepto de manera categórica y no 
Memorias del VII Encuentro Nacional de Experiencias en la Enseñanza de la Biología y la Educación Ambiental y 11 Congreso Nacional de Investigación en la Enseñanza de la Biología

integral en concordancia con los planteamientos de Giordan (1993). Por otro lado el caso 3 la estudiante presenta dificultades en la comprensión del concepto de biodiversidad "la biodiversidad está relacionado entre diferenciar casa y también se trata cono en las relaciones sexuales y ps también como las diferencias entre plantas objetos entre ciudadanos y animales". Ya en relación a las causas de la pérdida de la biodiversidad, estos casos reconocen algunas muy ligadas a la intervención antropica coincidiendo con los planteamientos de Martínez (2002) como: caso 1 "en algunos porque acaban su medio ambiente o algunas personas encuentran algún valor ya sea su carne piel colmillos y las matan para ganar dinero" y caso 5 "porque destruyen los hábitats naturales como la tala de árboles, deforestación ó se extinguen por la cazería" pero aun no reconocen la introducción de plantas exóticas como una causa, también el caso 3 demuestra que no reconoce ninguna de las causas de la pérdida de la biodiversidad, esto en relación con los niveles de ingenuidad planteados también por de Longhi (2006). Ahora se demostrará el proceso de complejización de sus ideas previas.

Teniendo en cuenta que ninguno de los estudiantes reconoce la introducción de plantas exóticas como una problemática que incide en la pérdida de la biodiversidad, la docente demuestra por medio de una clase magistral una contextualización sobre esta problemática, que despierta el interés en los estudiantes para indagar la biodiversidad en su entorno por medio de la diferenciación de plantas exóticas y nativas.

Estudiante 1. 3, 5: estos estudiantes alcanzaron el nivel de principiante debido a que cada uno de ellos por medio de las actividades como las exposiciones, mapas conceptuales y la indagación de las diferencia entre seres vivos entre ellos plantas de procedencia exóticas y nativas en el patio del colegio, lograron acercamientos al concepto de especie: "hay muchas especies de seres vivos y se diferencian por sus características como la apariencia de este ser o para ser más específicos su $A D N$ ", "especies es que en un campo donde alla diferentes plantas, arboles, animales ejemplo una araña un cucarron nose pueden reproducir porque no son la misma especie. Porque no tienen el mismo ADN, osea la misma cantidad de cromosomas", estos acercamientos denotan que los estudiantes presentan dificultades en la comprensión del concepto de especie, ya que se confunde este concepto con el de población y comunidad basados en la simple observación, esto en concordancia con los planteamientos de adeniyi 1985; Berzal de Pedrazzini y Barberá 1993; Develay y Ginsburger-Vogel; 1986; Driver et al 1989; Pozo et al 1999; Serrano y Blanco 1988 citados por Jiménez Tejada (2009). No obstante, en la indagación de la biodiversidad en el patio de su colegio los estudiantes encontraron que plantas conocidas como el holly, el pino, el eucalipto y las acacias son exóticas y plantas como el lulo, el hayuelo, y el chicala son nativas; por medio de esto la estudiante 1 reconoce algunos efectos de la introducción de plantas exóticas como "en el pino sus frutos no son comestibles para otros animales", en coherencia con Martínez (2002) y con ello logra acercamientos al nivel de aprendiz, los casos 3 y 5 reconocen la problemática pero no generan reflexiones ó reconocimientos explícitos sobre las consecuencias referentes a esta 
Memorias del VII Encuentro Nacional de Experiencias en la Enseñanza de la Biología y la Educación Ambiental y 11 Congreso Nacional de Investigación en la Enseñanza de la Biología

problemática. Teniendo en cuenta lo mencionado sobre estos casos se puede decir que su comprensión sobre biodiversidad se acerca al concepto de variedad de seres vivos en un ecosistema de acuerdo con Crisci (2006) y a su vez presentan dificultades para reconocer y analizar la problemática mencionada anteriormente.

Estudiantes 2, 4, 6: en este caso los tres estudiantes alcanzaron el nivel de aprendiz con algunos acercamientos al nivel de maestría (estudiante 4). Por medio de las mismas actividades que mencionamos en los casos anteriores, los estudiantes se acercan al concepto de especie demostrando las mismas dificultades ya que también lo confunden con el de población y comunidad basados en la simple observación (estudiantes 2) en concordancia con los autores mencionados anteriormente y a su vez citados por Jiménez Tejada (2009), pero en el caso de los estudiantes 4 y 6 la definición de especie planteada: "características que hay entre los genotipos de los animales que hace que se diferencien" demuestra acercamientos a la definición de especie genética en coherencia con Uribeondo (2007). Por otro lado, los estudiantes a través de la socialización de las actividades, trabajos extraescolares, los debates realizados en clase, las plantas exóticas y nativas encontradas en el patio del colegio, que se mencionaron anteriormente, reconocen la influencia de los factores abióticos en el ecosistema y la biodiversidad "allá es muy húmedo y hay más posibilidades que hayan mas animales que acá en el colegio porque el suelo es bastante seco" (estudiante 4) "en el lugar en donde nosotros estuvimos hay una buena luz y poca en el colegio (...) allá habían muchas más flores y plantas" (estudiante 6), y generan reflexiones sobre los efectos de las especies de plantas exóticas sobre especies de plantas nativas al mencionar "las plantas exóticas pueden roban nutrientes de otras plantas" (estudiante 2), "este tipo de plantas como el eucalipto como que consumen mucha agua y secan el suelo" (estudiante 4) "ya no se conservan las características de nuestros ecosistemas", afirmaciones que coinciden con los planteamientos de Martínez (2002), esto indica a su vez una comprensión por parte de los estudiantes del concepto de biodiversidad, no solo en el número de especies, sino también las funciones que ejercen los seres vivos con su medio físico en acercándose a las afirmaciones de Crisci (2006) sobre el nivel ecosistémico y Noss (1990) en el atributo de función.

A partir de ello estos estudiantes comienzan a aportar soluciones a esta problemáticas presentes en el patio de su colegio, donde proponen la reorganización de este espacio con ayuda de la comunidad académica y la siembra de nuevas especies de plantas nativas en la huerta escolar, como una forma también de conservar la biodiversidad presente en su colegio, de acuerdo con Orozco y Soto (2013).

Dimensión de comunicación: Durante el desarrollo de esta unidad didáctica se observan avances en la intención de la oralidad y la preocupación de los 
Memorias del VII Encuentro Nacional de Experiencias en la Enseñanza de la Biología y la Educación Ambiental y 11 Congreso Nacional de Investigación en la Enseñanza de la Biología

estudiantes por comunicar lo aprendido y las reflexiones construidas en torno a la problemática de los impactos de la introducción de especies exóticas.

Durante las ideas previas, Los estudiantes 1, 3, 4, 5, 6 se ubicaron en el nivel de ingenuo debido a que estos presentaba ideas poco claras sobre el concepto de biodiversidad como: "la biodiversidad está relacionada entre diferenciar cosas y se trata como en las relaciones sexuales y pues también como diferencias entre plantas objetos, ciudades y animales" (estudiante 3). Por otro lado también presentaron manejo de solo un sistema de símbolos como los ejemplos: "es todo lo relacionado con la naturaleza y vida ejemplo: animales y plantas" (estudiante 4, 5). Y por último los estudiantes 1, 3, 6 no presentan intención de comunicación oral y estética. En cambio la estudiantes 2 se ubico en el nivel principiante debido expresa sus ideas de manera clara aunque con falta de profundidad sobre el concepto de biodiversidad, la biodiversidad en su entorno, y la problemática a tratar durante la unidad: "en ciudad bolívar hay poca naturaleza, solo hay basura perros y humanos en comparación y a comparación en la naturaleza hay animales", todo ello teniendo de acuerdo a los planteamientos de Stone (1999) sobre los niveles de comprensión para esta dimensión.

En el transcurso de las actividades desarrolladas durante la unidad, la estudiante 3 permanece en el nivel ingenuo, el estudiante 5 alcanza en nivel de principiante debido a que Evidencia complejización de las ideas previas por medio de la realización de mapas conceptuales, la indagación en el patio del colegio pero estas se desarrollan de manera muy ceñida a las indicaciones del maestro y para dar explicaciones o argumentar ahora usa el símbolo de las analogías para hacerse entender "la especie es como las marcas de carros, usted ve que cada marca de carro tiene unas características propias".

Los estudiantes 1, 2, 4, 6, alcanzan el nivel de aprendiz, los 4 casos demuestran la complejización de sus ideas previas por medio de la realización y sustentación de mapas conceptuales, carteleras, e indagación en el patio del colegio, donde cada uno de ellos demuestra preocupación por comunicar de manera clara sus ideas, utilizar diferentes géneros con sus respectivas pautas para dar a conocer el conocimiento aprendido sobre biodiversidad, y la problemática a tratar durante la unidad. La estudiante 1 expresa: "la idea es presentar la planta nativa, mostrar fotografías, su descripción, y como se siembra" "la idea profe, es sembrarla y anotar todo el proceso de crecimiento". La estudiante 2 Demuestra la preocupación por presentar el proyecto final por medio de un genero distinto (friso) siendo consciente de las pautas que implica, presentación de una introducción un desarrollo del trabajo con imágenes y descripciones y conclusiones contrastadas con definiciones teóricas, como la definición de biodiversidad según conservación internacional. Por otro lado los estudiantes 4 y 6 logran utilizar de género las exposiciones con sus respectivas pautas de manera consciente (utilización de imágenes y expresión oral adecuada ó muestras de imágenes y plantas), presenta también una intención de oralidad y estética al momento de presentar sus trabajos. Logran utilizar ahora un sistema 
Bio-grafia Escritos sobre la Biología y su Enseñanza.

Edición Extra-Ordinaria. ISSN 2027-1034 P. p122 - 132

Memorias del VII Encuentro Nacional de Experiencias en la Enseñanza de la Biología y la Educación Ambiental y 11 Congreso Nacional de Investigación en la Enseñanza de la Biología

de como el de realizar conclusiones por medio de comparaciones "allá es muy húmedo y hay más posibilidades que hayan animales de tierra que acá en el colegio porque el suelo es bastante seco".

\section{Conclusiones}

El reconocimiento de las plantas exóticas por medio de la indagación de la biodiversidad en el patio del colegio permitió que los estudiantes adquirieran mayor nivel de comprensión sobre el concepto de biodiversidad en la dimensión de contenido, ya que lograron pasar de la idea de modelo aditivo a un modelo de interacción, es decir, la variabilidad de seres vivos y las funciones que estos ejercen con su medio físico; así mismo en la dimensión de comunicación adquirieron intencionalidad oral, estética al mencionar soluciones con respecto a las desventajas que proveen las plantas exóticas en el medio, además adquirieron mayor flexibilidad para utilizar diferentes géneros y símbolos como la realización de trabajos en grupo como carteleras, exposiciones, frisos, debates, utilización de ejemplos, analogías y mapas conceptuales de manera apropiada.

\section{Bibliografía}

Ángel, G y De Longhi, A. (2006). Propuesta curricular de hipótesis de progresión para conceptos estructurantes de ecología. Campo abierto, 25,2, pp 13-48.

Arnal, J. \& Latorre, A. (1992). Naturaleza de la Investigación Educación, Investigación Educativa: Fundamentos y Metodologías, Barcelona: Labor, pp. 24 - 49.

Boix, V \& Gardner H, en Stone (1999). Enseñanza para la comprensión: Vinculación entre la investigación y la práctica. Buenos Aires: Paidós, p. 441

Crisci, J. (2006). Espejos de nuestra época: Biodiversidad, Sistemática y Educación. Gayana Bot. 63(1), 106-114.

García, Cano y Rodríguez (2003) ¿qué ecología enseñar? La perspectiva del mesocosmos: la construcción de las nociones de biodiversidad y de relación ecológica. Investigando el ecosistema. Ed Sevilla: Díada. p 51.

Giordan A. (1993). Estructuración 2: la adquisición de conocimientos. La enseñanza de las ciencias siglo XXI. Español, pp.149-180.

Martínez, J. (2002) La Evolución y la conservación de la biodiversidad. En M. Soler (Ed). Evolución la base de la biología. España: Editorial Proyecto Sur (pp 408-416).

Orozco Y. \& Soto R. (2013). Msc. Lídice Castro Serrano. IX congreso de educación ambiental para el desarrollo sostenible. La habana, Cuba.

Rozzi, R., Feinsinger P., Massardo, F. \& Primack, R. (2001). Qué es la diversidad biológica. En R. Primack, R. Rozzi, P. Feinsinger \& R. Dirzo (Comps) Fundamentos de Conservación Biológica. Perspectivas Latinoamericanas. México D.F: Fondo de Cultura Económica. 
Bio-grafia Escritos sobre la Biología y su Enseñanza.

Edición Extra-Ordinaria. ISSN 2027-1034 P. p122-132

Memorias del VII Encuentro Nacional de Experiencias en la Enseñanza de la

Biología y la Educación Ambiental y II Congreso Nacional de Investigación en la Enseñanza de la Biología

Noss, R. (1990). Indicators for monitoring biodiversity. A hierarchical model. Conservation biology, 4: 355-364.

Stone, M (1999) ¿Qué es la enseñanza para la comprensión? En: Stone, Martha. La enseñanza para la Comprensión: Vinculación entre la investigación y la práctica. Buenos Aires: Paidós, p. 441.

Tejada, M (2009). Los conceptos de población y de especie en la enseñanza de la biología: concepciones, dificultades y perspectivas. (Tesis Doctoral). Universidad de Granada.

Uribeondo, J. D. (2007). Biodiversidad El Mosaico de la Vida. En J. D.Uribeondo, Biodiversidad El Mosaico de la Vida (págs. 31-41). España: Fundación Española para la Ciencia y la Tecnología. 